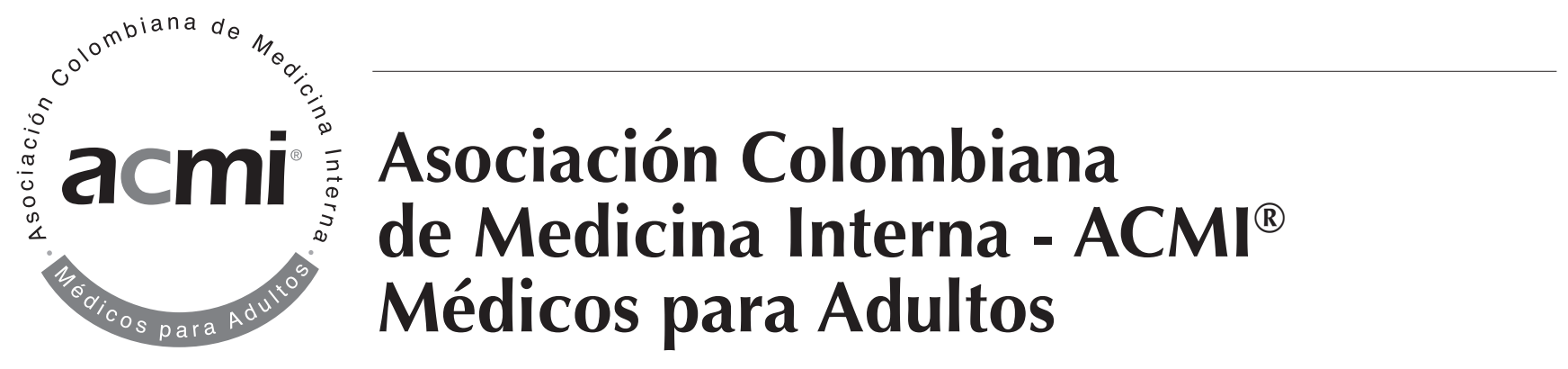

\title{
Junta Directiva Nacional 2014-2016
}

\section{Presidente}

Helí Hernández Ayazo

Secretario Ejecutivo

Virgil Carballo Zárate

\section{Tesorero}

Yazmín Abuabara Turbay

Fiscal

José Javier Arango Álvarez

\section{Presidentes Capítulos}

Alto Magdalena

Homero Puello Galarcio

Antioquia-Chocó

José Ignacio Tobón Acosta

Caldas

Edgar Eduardo Castro Osorio

Caribe

William Sánchez Rincones

Central

Análida Elizabeth Pinilla Roa

\section{Costa Atlántica}

Angelo María Alario Bello

\section{Morrosquillo}

Tatiana Espinosa Espitia

Nariño

Norte de Santander

Fredy Niño Prato

Quindío

Yorlady Uchamocha Pérez

Risaralda

Tatiana Álvarez Vera

Santander

Gustavo Adolfo Parra Zuluaga

Valle-Cauca

María Eugenia Casanova Valderrama
Ex presidentes

Rafael Carrizosa ${ }^{+}$

Roberto De Zubiría +

Gustavo Montejo ${ }^{+}$

Adolfo De Francisco

William Rojas

Eduardo De Zubiría

Hernando Sarasti

Roso Alfredo Cala ${ }^{+}$

Fernando Chalem ${ }^{+}$

Pérez Starusta

José María Mora ${ }^{+}$

Alberto Restrepo ${ }^{+}$

Roberto Esguerra

Jaime Campos ${ }^{+}$

Darío Maldonado

Javier Molina López

Hernando Matiz

Adolfo Vera

Roberto D'Achiardi

Guillermo Vanegas

Dora Inés Molina de Salazar

Gustavo Márquez

María Nelly Niño de Arboleda

Jairo Roa

Gregorio Sánchez

Juan Manuel Senior

Natalia Londoño 


\section{Anuncios ACMI}

\section{Congreso ACMI-ACP Nuevos Desafíos en la Práctica Clínica 14-16 de agosto de 2015 • Montería, Córdoba}

\section{Programa Científico Preliminar}

Sesión Plenaria. Cefaleas • Las horas doradas de la enfermedad vascular cerebral.

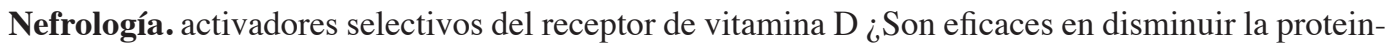
uria? • Hiperkalemia: resina de intercambio-Quelantes • Riñón bioartificial.

Diabetes. Actualización en cetoacidosis • Hipoglucemias • Antidiabéticos y RCVG.

Cardiología. Diagnóstico de FA en ACV criptogénico • Triple terapia • Terapia Dual ¿Cuánto tiempo?

Práctica Médica. Perlas éticas y jurídicas de la práctica médica • ACSC ¿Qué y para qué? • Ley fundamental de salud: cambios en la práctica médica $\bullet$ Recertificación en Medicina Interna • El ABC de la exacerbación de la EPOC • Asma en urgencias • Nuevos métodos Dx en TBC.

Diabetes. Nuevas tecnologías en diabetes, dispositivos biomédicos $\bullet$ Monitoreo continuo de glucosa - Glucómetros.

Cardiología. Biomarcadores cardiacos en la emergencia $\bullet$ Síndrome coronario agudo $\bullet$ Taquiarritmias - Taquiarritmias en urgencias $\bullet$ Nuevos anticoagulantes orales en FA $\bullet$ Nuevos anticoagulantes orales: más allá de la anticoagulación en FA • MAPA en el diagnóstico de HTA.

Pie Diabético. ¿Qué es el pie diabético? • Enfermedad arterial periférica y polineuropatía diabética como componente del pie diabético $\bullet$ Pié diabético infectado.

Endocrinología. Hipotiroidismo subclínico • Insulinización en el paciente diabético hospitalizado - Nódulo tiroideo.

Infectología. Síndrome ícterohemorrágico • Nefritis lúpica y su impacto en la región caribe colombiana $\bullet$ Epidemias modernas, no más ébola, piensa en chagas •

HTA. Crisis hipertensiva $\bullet$ Falla cardiaca aguda $\bullet$ Manifestaciones cardiovasculares de las enfermedades reumatológicas.

Gastroenterología. Nuevas perspectivas en el manejo del hígado graso $\bullet$ Diarrea crónica $\bullet$ Enfermedad por reflujo gastroesofágico.

Hematología. Enfoque diagnóstico de la gamapatía monoclonal • Urgencias oncológicas • Diagnóstico de cáncer con primario desconocido.

Simposio SOLAMI. Medición del efecto anticoagulante con los nuevos anticoagulantes orales $\bullet$ Son todos los betabloqueadores iguales, un efoque práctico $\bullet$ Colesterol y memoria metabólica.

Cardiología. Falla cardiaca crónica • Hipertensión pulmonar • Dispositivos en falla cardiaca.

Simposio Acta Médica Colombiana. Historia de Acta Médica Colombiana • Normas de publicación en revistas biomédicas $\bullet$ Cómo citar referencias bibliográficas $\bullet$ Guías mínimas para reportar estudios aleatorizados (consort) y revisiones sistemáticas y metaanálisis (Prisma) • Conflicto de intereses en publicaciones biomédicas • Consideraciones éticas en publicaciones biomédicas.

Educación médica. Rescatando la clínica -Experiencia con aprendizaje basado en problemas • Innovación curricular para forjar el orgullo de ser internista- ¿Qué es el internista latinoamericano? - Medicina interna hospitalaria • Aspectos básicos de la calidad de la atención médica.

Obesidad. Tratamiento farmacológico • Tratamiento quirúrgico, visión del internista $•$ Manejo posbariátrico. 


\section{Congreso ACMI-ACP. Nuevos Desafíos en la Práctica Clínica}

\section{Programa Científico Preliminar $($ (Continuación)}

Dislipidemias. RCVG • Inhibición de la PSCK9, el inicio de una nueva era para controlar hipercolesterolemia y lograr metas • Terapia no estatínica.

Imagenología. Neuroimangen ¿cuándo resonancia, cuándo tomografía? • Fibrosis miocárdica: utilidad de la resonancia magnética cardiaca- El dolor lumbar: el papel de las imágenes • Tomografía multicorte en litiasis ureteral (Urotac).

Dermatología. Las ocho dermatosis que el internista debe conocer • Tiroides y piel • Reacción medicamentosa.

Simposio SOLAMI 2. Urgencias críticas en la madrugada de una fiesta Rave • El vino y el corazón - Primera convulsión en el adulto, Abordaje del internista.

Sesión Plenaria. Nueva epigenética de la enfermedad cardiovascular • Causas frecuentes de hiponatremias mortales.

Simposio de interés especial. Enfermedad de Fabry • Urolitiasis ¿Cuál es el rol del intersnita? •ASA y vitamina $\mathrm{D}$ en cáncer de colon.

VIH • SIDA. Coinfección HIV - Hepatitis B y C • Nuevos medicamentos en VIH • Riesgo cardiovascular en VIH.

Simposio de interés especial 2. Efectos metabólicos del ejercicio $\bullet$ Nefroprotección con medicamentos para la diabetes ¿Cuál es la teoría y qué evidencia tenemos? • Enfermedad tromboembólica.

Historia de la Medicina. Historia de la Potra $\bullet$ Papel de los protohospitales coloniales y la legislación médica •Eusebio Vargas Vélez, docente de la Universidad de Cartagena ¿Cómo hacer historia?.

Simposio LASH. Qué nos ha diseñado el PURE en Latinoamérica • Enfoque terapéutico de la HTA, Cuándo dar terapia combinada $\bullet$ Complicaciones agudas de la HTA.

Embarazo y condiciones médicas. Hipertensión y embarazo • Diabetes y embarazo • Tiroides y embarazo • Bacteriuria asintomática en ancianos • Infección recurrente de vías urinarias en mujer joven $\bullet$ IVO asociada al cuidado de la salud y a catéter vesical.

Reumatología. Fibromialgia, mito • Artritis reumatoidea: utilidad de nuevos biomarcadores $\bullet$ LES: actualización en el tratamiento.

Sesión Plenaria. El papel del internista en el síndrome de boca urente • Experiencia con el Chikungunya.

\section{Inscripciones}

Miembros ACMI ${ }^{\circledR}$ a paz y salvo $\ldots \ldots \ldots \ldots \ldots \ldots \ldots \ldots \ldots$ sin costo

Miembros ACP a paz y salvo $\ldots \ldots \ldots \ldots \ldots \ldots \ldots \ldots \ldots \ldots \ldots \ldots \ldots$ sin costo

Miembros de Sociedades . . . . . . . . . . . . . . . . . . . \$400.000

Médicos generales y otros profesionales de la salud $\ldots \ldots \ldots \ldots \$ 150.000$

Estudiantes $\ldots \ldots \ldots \ldots \ldots \ldots \ldots \ldots \ldots \ldots \ldots \ldots \ldots \ldots \ldots \ldots \ldots \ldots \ldots$

\section{Informes:}

Asociación Colombiana de Medicina Interna

Tels. (57-1) 2368682- 2368994 • Cel. 310-465 3637 • Fax 2187860

acmi@acmi.org.co•acminal@acmi.org.co•gerente.acmi@acmi.org.co

www.acmi.org.co 